\title{
Estimation of Power Function Distribution Based on Selective Order Statistic
}

\author{
Mohd T. Alodat ${ }^{1} \quad$ Mohammad Y. Al-Rawwash $^{2}$ \\ Sameer A. Al-Subh ${ }^{3}$
}

\begin{abstract}
In this article, we present the selective order statistic sampling scheme as a promising approach to estimate the parameter of the univariate power function distribution. We derive the maximum likelihood estimator and the method of moments estimator of the power function distribution parameter as well as the reliability parameter and the ratio of two means. Moreover, we derive the asymptotic properties of the proposed estimators. Finally, we conduct simulation studies to investigate the performance of the selective order statistic scheme and concluded that it suits the power function distribution and we found that the maximum likelihood estimator is better than the method of moments estimator under the selective order statistic sampling scheme.
\end{abstract}

\section{Introduction}

The power function distribution (PFD) is one of the most common distributions used to model real data sets in different areas including but not limited to lifetime, income, industry and environment. Many researchers investigated the characteristics of the PFD and showed how handy and flexible this distribution is compared to the list of well known distributions such as exponential, Weibull, gamma, lognormal, etc. Menconi (1995) proposed using a simple model to find failure rate and reliability figures and found that the models based on PFD are applicable, simple and flexible compared to other distributions such as lognormal and Weibull. Menconi and Bary (1996) concluded that the PFD is more handy and flexible alternative compared to more complex models used to measure the reliability of electrical components. A major challenge that encounters researchers in lifetime, industry, environment and even agriculture is the sampling and experimental cost of data for the subjects of interest. In addition, the expensive nature of the experiment urges researchers to consider effective sampling schemes to pursue their missions. In certain fields of research, data collection of certain variables of interest is expensive, difficult to achieve and time-consuming task while ranking the items without actual quantifications is an affordable mission. The ranked set sampling (RSS) scheme proposed by

\footnotetext{
${ }^{1}$ Department of Statistics, Yarmouk University, Irbid, Jordan; alodatmts@yahoo.com

${ }^{2}$ Department of Statistics, Yarmouk University, Irbid, Jordan; rawwash@yu.edu.jo

${ }^{3}$ Department of Mathematics \& Statistics, Mutah University, Karak, Jordan; salsubh@yahoo.com
} 
McIntyer (1952) is a promising sampling approach that is proved to increase efficiency and reduce the cost. Depending on visual ranking rather than actual quantification of the units, an RSS of size can be obtained as follows. First, we select $m$ independent random samples each of size $m$ from the population of interest. Second, the $j$-th order statistic of the $j$-th sample can be detected visually or by any crude method and the selected unit will be chosen for actual quantification. The process may be repeated $r$-times to obtain an RSS of size $n=r m$. Although RSS has been used as a sampling technique for estimating the characteristics of a nonparametric population, several authors showed that the accuracy can be improved under parametric setups. Also, they showed that simple random sample (SRS) fail to produce an efficient estimator of the population mean compared to RSS and its significant modifications (Halls and Dell, 1966; Dell and Clutter, 1972; Stokes and Sager, 1988; Muttlak, 1997; Alodat and Al-Saleh, 2001; Chen et al., 2004 and Alodat et al., 2010). McIntyer (1952) main goal of RSS scheme was directed to estimate the population mean for a nonparametric problem. Later Takahasi and Wakimoto (1968) showed that the sample mean based on an RSS is the minimum variance unbiased estimator of the population mean. Also they concluded, based on SRS and RSS of the same size, that the relative efficiency of the sample mean using RSS with respect to SRS is bounded between 1 and $\frac{m+1}{2}$. RSS is a very useful technique when relying on ranking units through visual inspection leading to negligible cost.

The selective order statistic (SOS) works selectively depending on the amount of information available in the data set. First, we note that the total number of possible sampling schemes is $\left(\begin{array}{c}2 m-1 \\ m\end{array}\right)$ which can be used to select a sample from the population of interest by the means of visual ranking. These schemes are denoted by

$$
\mathcal{S}_{m}=\left(j_{1}, \ldots, j_{m}\right): j_{i}=1, \ldots, m ; i=1, \ldots, m
$$

where the $m$-tuple $\left(j_{1}, \ldots, j_{m}\right) \in \mathcal{S}_{m}$ mean that the unit of order $j_{i}$ is selected from the $i$-th set, $i=1, \ldots, m$. For a specific regular parametric family, we seek the sampling scheme among those in $\mathcal{S}_{m}$ that collects the largest amount of Fisher's information about the parameter $\theta$. To do so, we define $Y_{j: m}$ to be the $j$-th order statistic obtained from the SRS of size $m$ and let $I_{Y_{j: m}}(\theta)$ denote the Fisher's information numbers based on $Y_{j: m}$. The SOS will be obtained as follows:

1. Find that value $j_{0} \in 1, \ldots, m$ such that $j_{0}=\underset{1 \leq j \leq m}{\arg \max } I_{Y_{j: m}}(\theta)$.

2. For each of the $m$ sets, we choose for actual measurement the unit with order $j_{0}$.

The rest of the article is organized as follows. In Section 2, we introduce the PFD and derive the Fisher information number of the $j$-th order statistic. Also we investigate the performance of RSS and SOS via their information numbers and identify the proper sampling scheme that suits the PFD. In Section 3, we derive the maximum likelihood estimators (MLE), the method of moments estimators (MOME) of the PFD parameter, the reliability parameter as well as the ratio for two PFD parameters. Moreover, we obtain the asymptotic distribution of the parameter estimates in addition to the large-sample confidence intervals. In Section 4, we conduct a simulation study to investigate the bias and mean squared errors (MSE) of the parameter estimates as well as the confidence interval width. Finally, we outline the proofs of the theory of the article in the appendix. 


\section{Power Function Distribution}

Let $X$ be a random variable distributed as PFD with shape parameter $\beta$ and scale parameter $\alpha$, then the probability density function (pdf) is given by

$$
f(x ; \alpha, \beta)=\frac{\beta x^{\beta-1}}{\alpha^{\beta}} ; \quad 0<x<\alpha, \alpha>0, \beta>0 .
$$

If we use the mean parameterization by taking the scale parameter $\alpha=1$ and $\beta=\frac{\theta}{1-\theta}$, then the pdf of the PDF will be

$$
f(x ; \theta)=\frac{\theta}{1-\theta} x^{\frac{2 \theta-1}{1-\theta}} ; \quad 0<x<1,0<\theta<1 .
$$

It is easy to check that the mean and the variance are $E(X)=\theta$ and $\sigma_{X}^{2}=\frac{\theta(1-\theta)^{2}}{2-\theta}$, respectively.

Also, the cumulative distribution function is $F(x)=x^{\frac{\theta}{1-\theta}}, 0<x<1$. The properties and merits of the PFD were studied in the literature to investigate the reliability of products and asses the performance of electrical components in industrial and electrical engineering (Meniconi, 1995 and Meniconi and Barry, 1996). The PFD is preferred over log normal, exponential and Weibull distributions since it fits the failure data sets more efficiently (Ahsanullah, 1989; Meniconi, 1995; Saran and Pandey, 2004 and Saleem et al., 2010). Choosing the appropriate sampling scheme depends on the setup of the experiment and the nature of the target population and it may occur in certain practical situations that SOS is more convenient than the traditional SRS and RSS. To investigate this claim and to make inference about PFD parameter, we find the pdf of $Y_{j: m}$ as follows:

$$
f_{Y_{j: m}}(y ; \theta)=C_{j, m} \frac{\theta}{1-\theta} y^{\frac{\theta(j+1)-1}{1-\theta}}\left(1-y^{\frac{\theta}{1-\theta}}\right)^{m-j},
$$

where

$$
C_{j, m}=\frac{m !}{(j-1) !(m-j) !}
$$

for $j=1,2, \ldots, m$.

Theorem 1. If $Y_{j: m}$ is the $j$-th order statistic with $p d f$ given in (2.2), then

$$
I_{Y_{j: m}}(\theta)=\frac{A_{j, m}}{\theta^{2}(1-\theta)^{2}},
$$

where

$$
A_{j, m}=C_{j, m} \int_{0}^{1}\left(1+j \log v-\frac{(m-j) v \log v}{1-v}\right)^{2} v^{j-1}(1-v)^{m-j} d v
$$

The proof of the theorem is outlined in the Appendix.

The term $A_{j, m}$ clearly shows that the value of $j$ maximizing $I_{Y_{j: m}}(\theta)$ does not depend on $\theta$, hence it suffices to maximize the quantity $I_{Y_{j: m}}^{*}=\theta^{2}(1-\theta)^{2} I_{Y_{j: m}}(\theta)$ instead of $I_{Y_{j: m}}(\theta)$. However, the difficulty of this theoretical maximization leads to the numerical 
maximization as an alternative option. Table 1 shows the amount of information $\left(I^{*}\right)$ contained in the sample about the PFD parameter for different values of $j$ and $m$. It can be noticed from Table 1 that maximizing Fisher information number $I_{Y_{j: m}}(\theta)$, as a function of $j$, depends on the set size $m$. Our findings show that the amount of information about $\theta$ contained in the order statistic $Y_{j: m}$ attains its maximum at $j=j_{0}=1$, for $1 \leq m \leq 5$ and at $j=j_{0}=2$, for $6 \leq m \leq 10$. These results provide a sufficient evidence to collect our samples using the selective order statistic $Y_{j_{0}: m}$ when the underlying distribution is PFD with parameter $\theta$.

Table 1: The values of $I_{Y_{j: m}}^{*}$ for different values of $j$ and $m$

\begin{tabular}{rrrrrrrrr}
\hline$m$ & $j$ & $I_{Y_{j: m}}^{*}$ & $m$ & $j$ & $I_{Y_{j: m}}^{*}$ & $m$ & $j$ & $I_{Y_{j: m}}^{*}$ \\
\hline 2 & 1 & 1.808 & 6 & 4 & 2.920 & 9 & 1 & 5.472 \\
& 2 & 1.000 & & 5 & 1.984 & & 2 & 6.282 \\
3 & 1 & 2.500 & & 6 & 1.000 & & 3 & 6.123 \\
& 2 & 1.924 & 7 & 1 & 4.631 & & 4 & 5.558 \\
& 3 & 1.000 & & 2 & 5.004 & & 5 & 4.789 \\
4 & 1 & 3.111 & & 3 & 4.574 & & 6 & 3.910 \\
& 2 & 2.777 & & 4 & 3.831 & & 7 & 2.969 \\
& 3 & 1.960 & & 5 & 2.944 & & 8 & 1.993 \\
& 4 & 1.000 & & 6 & 1.988 & & 9 & 1.000 \\
5 & 1 & 3.662 & & 7 & 1.000 & 10 & 1 & 5.856 \\
& 2 & 3.569 & 8 & 1 & 5.065 & & 2 & 6.874 \\
& 3 & 2.875 & & 2 & 5.660 & & 3 & 6.850 \\
& 4 & 1.975 & & 3 & 5.365 & & 4 & 6.377 \\
& 5 & 1.000 & & 4 & 4.710 & & 5 & 5.673 \\
6 & 1 & 4.166 & & 5 & 3.880 & & 6 & 4.840 \\
& 2 & 4.309 & & 6 & 2.959 & & 7 & 3.930 \\
& 3 & 3.745 & & 7 & 1.991 & & 8 & 2.975 \\
& & & & 8 & 1.000 & & 9 & 1.994 \\
& & & & & & & 10 & 1.000 \\
\hline
\end{tabular}

Obtaining the Fisher information matrix is essential in estimation and it is part of the asymptotic distribution of the parameter estimator. On the other hand, deriving the confidence interval is vital in many applications and practical situations (Alodat et al., 2009; Al-Rawwash et al., 2010, and Al-Rawwash et al., 2014). To elaborate on the results in Table 1 , let $I_{S O S}^{(m)}(\theta)$ and $I_{R S S}^{(m)}(\theta)$ denote the amounts of information about $\theta$ contained in the SOS and RSS schemes respectively and define the relative information number based on the two schemes as follows:

$$
R I=\frac{I_{S O S}^{(m)}(\theta)}{I_{R S S}^{(m)}(\theta)} .
$$

Table 2 shows the values of $I_{S O S}^{(m)}(\theta), I_{R S S}^{(m)}(\theta)$ and $R I$ for $m=2, \ldots, 10$ that will be obtained based on the results of Table 1 . For example if $m=2$, then $I_{R S S}^{(2)}(\theta)=$ 
$1.80823+1=2.8082$ and $I_{S O S}^{(2)}(\theta)=2 \times 1.80823=3.6165$. The results in Table 2 clearly show that the SOS is more efficient than the RSS scheme in estimating the PFD parameter which is a motivation to adopt the SOS scheme.

Table 2: The values of $I_{S O S}^{(m)}(\theta), I_{R S S}^{(m)}(\theta)$, and RI for $m=2, \ldots, 10$

\begin{tabular}{rrrc}
\hline$m$ & $I_{R S S}^{(m)}(\theta)$ & $I_{S O S}^{(m)}(\theta)$ & $\mathrm{RI}$ \\
\hline 2 & 2.808 & 3.616 & 1.288 \\
3 & 5.424 & 7.500 & 1.383 \\
4 & 8.848 & 12.444 & 1.406 \\
5 & 13.082 & 18.310 & 1.400 \\
6 & 18.124 & 25.854 & 1.427 \\
7 & 23.972 & 35.028 & 1.461 \\
8 & 30.630 & 45.280 & 1.478 \\
9 & 38.096 & 56.538 & 1.484 \\
10 & 46.369 & 68.740 & 1.482 \\
\hline
\end{tabular}

\section{Estimation}

In this section we derive the MLE and the MOME of the PFD parameter based on the SOS scheme described in Section 2. In addition, we propose an estimator of the reliability parameter and discuss the asymptotic properties of the derived estimators. In addition, we will consider obtaining an SOS sample of size $n$ based on $n$ simple random subsamples each of size $m$.

\subsection{Estimating PDF parameter using MLE}

Theorem 2. Let $\mathbf{y}=\left(Y_{j_{0}: m^{\prime}}^{(1)}, Y_{j_{0}: m^{\prime}}^{(2)}, \ldots, Y_{j_{0}: m}^{(n)}\right)$ be an SOS sample obtained from PFD $(\theta)$. Also, let $\hat{\theta}_{M L E}$ be the MLE of $\theta$, then under certain regularity conditions we have

1. $\hat{\theta}_{M L E} \stackrel{P}{\rightarrow} \theta$

2. $\sqrt{n}\left(\hat{\theta}_{M L E}-\theta\right) \stackrel{D}{\rightarrow} N\left(0, I_{Y_{j_{0}: m}}^{-1}(\theta)\right)$

3. There is one unique MLE $\hat{\theta}_{M L E} \in(0,1)$

The proof of the theorem is outlined in the Appendix.

Now if we assume that $\hat{\theta}_{R S S, M L E}$ is the MLE of $\theta$ based on an RSS of $r$ cycles each of size $m$, we may compare $\hat{\theta}_{M L E}$ and $\hat{\theta}_{R S S, M L E}$ and show that $\sqrt{n}\left(\hat{\theta}_{R S S, M L E}-\theta\right) \stackrel{D}{\rightarrow}$ $N\left(0, I_{R S S}^{-1}(\theta)\right)$, where $I_{R S S}(\theta)=\frac{1}{m} \sum_{i=1}^{m} I_{Y_{i: m}}(\theta)$ and $n=r m$. Hence the asymptotic relative efficiency of $\hat{\theta}_{M L E}$ with respect to $\hat{\theta}_{R S S, M L E}$ is given by

$$
\operatorname{Aeff}\left(\hat{\theta}_{R S S, M L E}, \hat{\theta}_{M L E}\right)=\frac{I_{R S S}^{-1}(\theta)}{I_{Y_{j_{0}: m}}^{-1}(\theta)}=\frac{I_{Y_{j_{0}: m}}(\theta)}{\frac{1}{m} \sum_{i=1}^{m} I_{Y_{i: m}}(\theta)}
$$


Table 3: The efficiency values of $\hat{\theta}_{R S S, M L E}$ with respect to $\hat{\theta}_{M L E}$ for different values of $m$

\begin{tabular}{rc}
\hline $\mathrm{m}$ & Aeff $\left(\hat{\theta}_{R S S, M L E}, \hat{\theta}_{M L E}\right)$ \\
\hline 2 & 1.288 \\
3 & 1.383 \\
4 & 1.406 \\
5 & 1.400 \\
6 & 1.427 \\
7 & 1.461 \\
8 & 1.478 \\
9 & 1.484 \\
10 & 1.482 \\
\hline
\end{tabular}

Note that $\operatorname{Aeff}\left(\hat{\theta}_{R S S, M L E}, \hat{\theta}_{M L E}\right)$ is free of $\theta$ and its values are more than 1 for all $m$. Table 3 shows the values of Aeff $\left(\hat{\theta}_{R S S, M L E}, \hat{\theta}_{M L E}\right)$ for different $m$ where the MLE based on SOS is more efficient that the MLE based on RSS when estimating the PFD parameter. Hereafter, we carry out the estimation procedure based on SOS sampling scheme and we adopt the notation $\hat{\theta}_{M L E}$ and $\hat{\theta}_{M O M}$ to represent the maximum likelihood estimator and the method of moments estimator of $\theta$, respectively.

\subsection{Estimating the Reliability Parameter}

Discussing the quantity $\mathcal{R}=P(X<Y)$ is important in reliability analysis where $X$ and $Y$ are two independent random variables. If $X$ and $Y$ have power function distributions $\operatorname{PFD}\left(\theta_{1}\right)$ and $\operatorname{PFD}\left(\theta_{2}\right)$, respectively, then the true value of $\mathcal{R}$ represents a major tool in industrial applications. As an example, we may consider $X$ to be the stress value of a randomly selected device while $Y$ represents the strength of the device. In such a case, $\mathcal{R}$ represents the probability that the selected device is functioning successfully. For more details and interpretations concerning the values of $\mathcal{R}$, see Masoom and Woo (2005) and the references therein. In this section we derive an estimator of $\mathcal{R}$ when $X \sim \operatorname{PFD}\left(\theta_{1}\right)$ and $Y \sim \operatorname{PFD}\left(\theta_{2}\right)$, independently. Depending on the PFD assumption, we get the following

$$
\mathcal{R}=\frac{\theta_{2}\left(1-\theta_{1}\right)}{\theta_{1}+\theta_{2}-2 \theta_{1} \theta_{2}} .
$$

Using the invariance property, the MLE of $\mathcal{R}$ will be

$$
\hat{\mathcal{R}}_{M L E}=\frac{\hat{\theta}_{2, M L E}\left(1-\hat{\theta}_{1, M L E}\right)}{\hat{\theta}_{1, M L E}+\hat{\theta}_{2, M L E}-2 \hat{\theta}_{1, M L E} \hat{\theta}_{2, M L E}}
$$

where $\hat{\theta}_{1, M L E}$ and $\hat{\theta}_{2, M L E}$ are the MLE estimators of $\theta_{1}$ and $\theta_{2}$, respectively.

Theorem 3. Let $\mathbf{x}=\left(X_{j_{0}: m}^{(1)}, X_{j_{0}: m}^{(2)}, \ldots, X_{j_{0}: m}^{(n)}\right)$ and $\mathbf{y}=\left(Y_{j_{0}: m}^{(1)}, Y_{j_{0}: m}^{(2)}, \ldots, Y_{j_{0}: m}^{(n)}\right)$ be two independent SOS samples obtained from PFD $\left(\theta_{1}\right)$ and $P F D\left(\theta_{2}\right)$, respectively. Then, under certain regularity conditions we have 
1. $\hat{\mathcal{R}}_{M L E}$ is a consistent estimator of $\mathcal{R}$

2. $\sqrt{n}\left(\hat{\mathcal{R}}_{M L E}-\mathcal{R}\right) \stackrel{D}{\rightarrow} N\left(0, \sigma_{\hat{\mathcal{R}}_{M L E}}^{2}\right)$,

where

$$
\sigma_{\hat{\mathcal{R}}_{M L E}}^{2}=\frac{2 \theta_{1}^{2} \theta_{2}^{2}\left(1-\theta_{1}\right)^{2}\left(1-\theta_{2}\right)^{2}}{A_{j, m}\left(\theta_{1}+\theta_{2}-2 \theta_{1} \theta_{2}\right)^{4}}
$$

Remark. Theorem 3 allows us to conclude that $\sqrt{n} \frac{\mathcal{R}_{M L E}-\mathcal{R}}{\hat{\sigma}_{\hat{\mathcal{R}}_{M L E}}} \stackrel{D}{\rightarrow} N(0,1)$ which can be utilized to obtain a large-sample confidence interval for the parameter $\mathcal{R}$. hence a large sample $95 \%$ confidence interval for $\mathcal{R}$ is $\hat{\mathcal{R}}_{M L E} \pm 1.96 \sqrt{\frac{\hat{\sigma}_{\hat{\mathcal{R}}_{M L E}}}{n}}$.

\subsection{Method of Moment Estimators}

To derive the MOME for both parameters $\theta$ and $\mathcal{R}$, we rely on the results in Table 1 and assume that $j=1$ and 2, then we find the expected value of the $j$-th order statistic based on the $\operatorname{PFD}(\theta)$ as follows:

$$
\begin{aligned}
E\left(Y_{j: m}\right) & =C_{j, m} \frac{\theta}{1-\theta} \int_{0}^{1} y^{\frac{(j+1) \theta-1}{1-\theta}+1}\left(1-y^{\frac{\theta}{1-\theta}}\right)^{m-j} d y \\
& =C_{j, m} \frac{\theta}{1-\theta} \int_{0}^{1} y^{\frac{j \theta}{1-\theta}}\left(1-y^{\frac{\theta}{1-\theta}}\right)^{m-j} d y
\end{aligned}
$$

Setting $\nu=y^{\frac{\theta}{1-\theta}}$, we get

$$
\begin{aligned}
E\left(Y_{j: m}\right) & =C_{j, m} \int_{0}^{1} \nu^{\frac{1}{\theta}+j-2}(1-\nu)^{m-j} d \nu \\
& =C_{j, m} \Gamma(m-j+1) \frac{\Gamma\left(\frac{1}{\theta}+j-1\right)}{\Gamma\left(\frac{1}{\theta}+m\right)} \\
& = \begin{cases}\frac{m ! \theta^{m}}{\prod_{i=0}^{m-1}(i \theta+1)}, & \text { if } j=1 \\
\frac{m ! \theta^{m-1}}{\prod_{i=0}^{m-1}(i \theta+1)}, & \text { if } j=2\end{cases}
\end{aligned}
$$

Similarly it can be shown that the variance of $Y_{j: m}$ is

$$
V(\theta)=\frac{m ! \Gamma\left(\frac{2}{\theta}+j-2\right)}{(j-1) ! \Gamma\left(\frac{2}{\theta}+m-1\right)}-\left(\frac{m ! \Gamma\left(\frac{1}{\theta}+j-1\right)}{(j-1) ! \Gamma\left(\frac{1}{\theta}+m\right)}\right)^{2}
$$

Theorem 4. Let $Y_{j_{0}: m}^{(1)}, \ldots, Y_{j_{0}: m}^{(n)}$ be an SOS sample from PFD $(\theta)$ where $j_{0}=1$ or 2 and $m \leq 10$, then there exists a unique MOME for $\theta$.

Let $\hat{\theta}_{1, M O M}$ and $\hat{\theta}_{2, M O M}$ denote the MOME of $\theta_{1}$ and $\theta_{2}$, respectively, then the plug-in estimator of the reliability parameter based on MOME is given as follows:

$$
\hat{\mathcal{R}}_{M O M}=\frac{\hat{\theta}_{2, M O M}\left(1-\hat{\theta}_{1, M O M}\right)}{\hat{\theta}_{1, M O M}+\hat{\theta}_{2, M O M}-2 \hat{\theta}_{1, M O M} \hat{\theta}_{2, M O M}}
$$


Theorem 5. Let $\hat{\mathcal{R}}_{M O M}$ be the plug-in MOME of $\mathcal{R}$, then

1. $\hat{\mathcal{R}}_{M O M}$ is consistent estimator of $\mathcal{R}$.

2. $\sqrt{n}\left(\hat{\mathcal{R}}_{M O M}-\mathcal{R}\right) \stackrel{D}{\rightarrow} N\left(0, \mathcal{T}^{2}\left(\theta_{1}, \theta_{2}\right)\right)$

3. $\sqrt{n} \mathcal{T}^{-1}\left(\hat{\theta}_{1, M O M}, \hat{\theta}_{2, M O M}\right)\left(\hat{\mathcal{R}}_{M O M}-\mathcal{R}\right) \stackrel{D}{\rightarrow} N(0,1)$

where

$$
\mathcal{T}^{-1}\left(\hat{\theta}_{1, M O M}, \hat{\theta}_{2, M O M}\right)=\frac{1}{\mathcal{T}\left(\hat{\theta}_{1, M O M}, \hat{\theta}_{2, M O M}\right)}
$$

and

$$
\mathcal{T}^{2}\left(\theta_{1}, \theta_{2}\right)=\frac{V\left(\theta_{1}\right) K^{\prime 2}\left(\theta_{1}\right) \theta_{2}^{2}\left(1-\theta_{2}\right)^{2}+V\left(\theta_{2}\right) K^{\prime 2}\left(\theta_{2}\right) \theta_{1}^{2}\left(1-\theta_{1}\right)^{2}}{\left(\theta_{1}+\theta_{2}-2 \theta_{1} \theta_{2}\right)^{4}} .
$$

Accordingly, a large-sample $95 \%$ confidence interval for $\mathcal{R}$ is

$$
\hat{\mathcal{R}}_{M O M} \pm \frac{1.96}{\sqrt{n}} \times \mathcal{T}\left(\hat{\theta}_{1, M O M}, \hat{\theta}_{2, M O M}\right)
$$

\subsection{Estimating the Ratio of Two Means}

It is important in some applications to estimate the ratio of two population means which will be obtained in this article assuming that the data is selected from $\operatorname{PFD}\left(\theta_{1}\right)$ and $P F D\left(\theta_{2}\right)$. In addition, we carry out the estimation process using the MLE and the MOME as well to find the estimator of the population mean. To this end, we assume that $\hat{\theta}_{1, M L E}$ and $\hat{\theta}_{2, M L E}$ are the MLE estimators of $\theta_{1}$ and $\theta_{2}$, respectively and $\hat{\theta}_{1, M O M}$ and $\hat{\theta}_{2, M O M}$ are the MOME of $\theta_{1}$ and $\theta_{2}$, respectively. To estimate the ratio $\psi=\frac{\theta_{2}}{\theta_{1}}$, we present the following theorem.

Theorem 6. Consider the following two estimators of $\psi$ :

$$
\hat{\psi}_{M L E}=\frac{\hat{\theta}_{2, M L E}}{\hat{\theta}_{1, M L E}} \quad \text { and } \quad \hat{\psi}_{M O M}=\frac{\hat{\theta}_{2, M O M}}{\hat{\theta}_{1, M O M}} .
$$

Than

1. $\sqrt{n} \mathcal{J}^{-1}\left(\hat{\theta}_{1, M L E}, \hat{\theta}_{2, M L E}\right)\left(\hat{\psi}_{M L E}-\psi\right) \stackrel{D}{\rightarrow} N(0,1)$

2. $\sqrt{n} \mathcal{L}^{-1}\left(\hat{\theta}_{1, M O M}, \hat{\theta}_{2, M O M}\right)\left(\hat{\psi}_{M O M}-\psi\right) \stackrel{D}{\rightarrow} N(0,1)$

where

$$
\mathcal{J}\left(\theta_{1}, \theta_{2}\right)=\frac{\left(\theta_{2}^{2}\left(1-\theta_{1}\right)^{2}+\frac{\theta_{2}^{2}\left(1-\theta_{2}\right)^{2}}{\theta_{1}^{2}}\right)}{A_{j, m}}
$$

and

$$
\mathcal{L}\left(\theta_{1}, \theta_{2}\right)=\theta_{2}^{2} \theta_{1}^{-4} V\left(\theta_{1}\right) K^{\prime 2}\left(\theta_{1}\right)+\theta_{1}^{-2} V\left(\theta_{2}\right) K^{\prime 2}\left(\theta_{2}\right)
$$

The proof is similar to those of Theorem 4 and Theorem 5. 


\subsection{Pivotal Confidence Interval for $\theta$}

The asymptotic results obtained earlier provide us with a rigorous inferential approach for estimating $\theta, \mathcal{R}$, and $\psi$. As an alternative approach, we introduce pivotal quantities to obtain the confidence interval of $\theta$. To this end, we assume that $Y_{j_{0}: m}^{(1)}, Y_{j_{0}: m}^{(2)}, \ldots, Y_{j_{0}: m}^{(n)}$ are iid copies of the selective order statistic $Y_{j_{0}: m}$ and introduce the following pivotal quantity for $\theta$

$$
\begin{aligned}
F\left(Y_{j_{0}: m}^{(1)}, Y_{j_{0}: m}^{(2)}, \ldots, Y_{j_{0}: m}^{(n)} ; \theta\right) & =-2 \sum_{i=1}^{n} \log \left(F_{Y_{j_{0}: m}^{(i)}}\left(Y_{j_{0}: m}^{(i)} ; \theta\right)\right) \\
& =-2 \sum_{i=1}^{n} \log \left(\sum_{j=j_{0}}^{m}\left(\begin{array}{c}
m \\
j
\end{array}\right) Y_{j_{0}: m}^{(i) \frac{j \theta}{1-\theta}}\left(1-Y_{j_{0}: m}^{(i) \frac{\theta}{1-\theta}}\right)^{m-j}\right) .
\end{aligned}
$$

It can be shown that $F\left(Y_{j_{0}: m}^{(1)}, Y_{j_{0}: m}^{(2)}, \ldots, Y_{j_{0}: m}^{(n)} ; \theta\right)$ is a monotone increasing function in $\theta$ distributed as a chi-square with $n$ degrees-of-freedom. Hence there exists unique solutions for the equations

$$
F\left(Y_{j_{0}: m}^{(1)}, Y_{j_{0}: m}^{(2)}, \ldots, Y_{j_{0}: m}^{(n)} ; \theta\right)=\chi_{n, \frac{\alpha}{2}}^{2}
$$

and

$$
F\left(Y_{j_{0}: m}^{(1)}, Y_{j_{0}: m}^{(2)}, \ldots, Y_{j_{0}: m}^{(n)} ; \theta\right)=\chi_{n, 1-\frac{\alpha}{2}}^{2},
$$

where $\chi_{n, \frac{\alpha}{2}}^{2}$ is the $100\left(\frac{\alpha}{2}\right)$ quantile of the chi-square distribution with $n$ degrees-of-freedom. Since

$$
P\left(\chi_{n, \frac{\alpha}{2}}^{2}<F\left(Y_{j_{0}: m}^{(1)}, Y_{j_{0}: m}^{(2)}, \ldots, Y_{j_{0}: m}^{(n)} ; \theta\right)<\chi_{n, 1-\frac{\alpha}{2}}^{2}\right)=1-\alpha
$$

than a $100(1-\alpha) \%$ confidence interval of $\theta$ is

$$
\left(L_{\frac{\alpha}{2}}\left(Y_{j_{0}: m}^{(1)}, Y_{j_{0}: m}^{(2)}, \ldots, Y_{j_{0}: m}^{(n)}\right), U_{1-\frac{\alpha}{2}}\left(Y_{j_{0}: m}^{(1)}, Y_{j_{0}: m}^{(2)}, \ldots, Y_{j_{0}: m}^{(n)}\right)\right)
$$

where $L_{\frac{\alpha}{2}}\left(Y_{j_{0}: m}^{(1)}, Y_{j_{0}: m}^{(2)}, \ldots, Y_{j_{0}: m}^{(n)}\right)$ and $U_{1-\frac{\alpha}{2}}\left(Y_{j_{0}: m}^{(1)}, Y_{j_{0}: m}^{(2)}, \ldots, Y_{j_{0}: m}^{(n)}\right)$ are the solutions of $F\left(Y_{j_{0}: m}^{(1)}, Y_{j_{0}: m}^{(2)}, \ldots, Y_{j_{0}: m}^{(n)} ; \theta\right)=\chi_{n, \frac{\alpha}{2}}^{2}$ and $\left.F\left(Y_{j_{0}: m}^{(1)}, Y_{j_{0}: m}^{(2)}, \ldots, Y_{j_{0}: m}^{(n)}\right) ; \theta\right)=\chi_{n, 1-\frac{\alpha}{2}}^{2}$, for $\theta$, respectively. In the follwing section, we obtain the expected length of the confidence interval of $\theta$, i.e., $E\left(L_{F}\right)$ based on simulated data, where

$$
L_{F}=U_{1-\frac{\alpha}{2}}\left(Y_{j_{0}: m}^{(1)}, Y_{j_{0}: m}^{(2)}, \ldots, Y_{j_{0}: m}^{(n)}\right)-L_{\frac{\alpha}{2}}\left(Y_{j_{0}: m}^{(1)}, Y_{j_{0}: m}^{(2)}, \ldots, Y_{j_{0}: m}^{(n)}\right)
$$

\section{Simulation}

As an illustration, we conduct a simulation study to evaluate the performance of our proposed estimators and to assess the accuracy and efficiency of these estimators via the bias, mean squared errors as well as the expected length of the confidence intervals. To accomplish this, we simulate $n$ independent samples each of size $m$ from $\operatorname{PFD}\left(\theta_{1}\right)$ and simulate the same number of samples from $\operatorname{PFD}\left(\theta_{2}\right)$. Accordingly, the SOS scheme will operate as follows. Select one random sample of size $m$ say $X_{1}, \ldots, X_{m}$ from $\operatorname{PFD}\left(\theta_{1}\right)$ 
and sort the sample in an ascending order $X_{1: m} \leq \cdots \leq X_{m: m}$. Then, we choose the $j_{0}$-th order statistic, i.e., $X_{j_{0}: m}$ and repeat this process $n$ times to obtain an SOS sample from $\operatorname{PFD}\left(\theta_{1}\right)$. We follow the same strategy to generate an SOS sample from $\operatorname{PFD}\left(\theta_{2}\right)$ and the new set of SOS samples will be used to obtain the MLE and MOME via numerical approximation using a rich list of mathematical software. The Mathematica software function FindRoot is a convenient option that will be implemented to obtain our estimates. The procedure is repeated $N$ times to obtain a large number of the estimators of interest. The following algorithm is implemented:

1. We simulate two SOS samples each of size $n$ as explained earlier for different set size $m$.

2. We use the two SOS samples to obtain the values of $\hat{\theta}_{M L E}, \hat{\theta}_{M O M}, \hat{\mathcal{R}}_{M L E}, \hat{\mathcal{R}}_{M O M}$, $\hat{\psi}_{M L E}$, and $\hat{\psi}_{M O M}$.

3. We repeant steps 1 and 2 to get a random sample of size $N$ from the distribution of each estimator.

4. Let $\hat{\theta}$ denote an estimator of the parameter in (2.1) and let $\hat{\theta}_{(i)}, i=1,2, \ldots, N$, denote the values of the estimator $\hat{\theta}$ based on the $i$-th iteration, then the approximate bias $B(\hat{\theta})$ and the mean squared errors $M S E(\hat{\theta})$ are calculated as follows

$$
B(\hat{\theta}) \approx \frac{1}{N} \sum_{i=1}^{N}\left(\hat{\theta}_{(i)}-\theta\right) \quad \text { and } \quad M S E(\hat{\theta}) \approx \frac{1}{N} \sum_{i=1}^{N}\left(\hat{\theta}_{(i)}-\theta\right)^{2}
$$

where $\theta$ is the exact value of the parameter.

Similarly, the expected length of $95 \%$ confidence interval is calculated as follows

$$
E(L) \approx \frac{1}{N} \sum_{i=1}^{N}\left(\hat{U}_{i}-\hat{L}_{i}\right)
$$

where $\left(\hat{L}_{i}, \hat{U}_{i}\right)$ is the corresponding confidence interval estimate obtained in the $i$-th iteration. The above algorithm has been implemented for $N=10000, m=1,2, \ldots, 10$ and $n=10,20,30,40,50$. The results of the simulation study are presented in Tables 4-6. The results allow us to conclude the following:

1. It is clear that the MLE is better than the MOME in terms of the bias and the MSE values for all the parameters of interest.

2. The bias and MSE values decrease as $n$ increases.

3. Increasing the set size $m$ helps decreasing the MSE values.

4. The expected length of the confidence interval is getting smaller as $n$ increases.

5. Increasing the set size $m$ decreases the expected length of the confidence interval.

6. The expected length of the confidence interval is affected by the initial true value of $\theta$. 


\section{Conclusion}

In this article, we introduced the SOS as a potential sampling scheme to provide good estimators of the PFD parameter. The importance of PFD in industry, lifetime, engineering, environment and many research areas motivated this article to search for the best sampling scheme that suits such distribution compared to the traditional sampling schemes. In this article, we derived the MLE and MOME for the parameter of the PFD. Also we discussed the quantity $\mathcal{R}=P(X<Y)$ and derived its estimator using the MLE and MOME. Comparing two PFD parameters is important in many areas of application and therefore we explained the estimation strategy of the ratio of the two PFD parameters. We introduced the asymptotic results of the parameter estimators and derived the confidence interval of the PFD parameter. The results in Table 2 motivates us to use the SOS as a competitive scheme to carry on the estimation of the PFD parameter. Also, the simulation results support MLE as the best choice in terms of the MSE values compared to MOME.

\section{Acknowledgements}

The authors are grateful to an associate editor and to two anonymous referees for their valuable comments.

\section{References}

[1] Ahsanullah, M. (1989): Estimation of the parameters of a power function distribution by record values. Pakistan Journal of Statistics, 5(2), 189-194.

[2] Alodat, M. T. and Al-Saleh, M. F. (2001): Variation of ranked set sampling. Journal of Applied Statistical Science, 10(2), 137-146.

[3] Alodat, M. T., AL-Rawwash M. Y., and Nawajah, I. M. (2009): Analysis of simple linear regression via median ranked set sampling. Metron, 67(1), 57-74.

[4] Alodat, M. T., AL-Rawwash M. Y., and Nawajah, I. M. (2010): Inference about the regression parameters using median ranked set sampling. Communications in Statistics-Theory and Methods, 39(14), 2604-2616.

[5] Al-Rawwash, M., Alodat, M. T., Aludaat, K. M., Odat, N. and Muhaidat, R. (2010): Prediction intervals for characteristics of future normal sample under moving ranked set sampling. Statistica, 70(2), 137-152.

[6] Al-Rawwash, M., Alodat, M. T. and Nawajah, I. (2014): Fisher information of the regression parameters using median ranked set sampling. In: Proceeding of the 3rd International Conference on Mathematical Sciences, 983-989. American Institute of Physics Proceeding.

[7] Chen, Z., Bai, Z. and Sinha, B. (2004): Ranked-Set Sampling: Theory and Applications. New York, NY: Springer. 
[8] Dell, T. R. and Clutter, L. (1972): Ranked set sampling theory with order statistics background. Biometrics, 28, 545-553.

[9] Halls, L. S. and Dell, T. R. (1966): Trial of ranked set sampling for forage yields. Forest Science, 12(1), 22-26.

[10] Kurosh, A. (1972): Higher algebra. Moscow: Mir Publishers.

[11] Lehmann, E. L. and Casella, G. (1983): Theory of Point Estimation. New York, NY: Springer.

[12] Masoom, M. and Woo, J. (2005): Inference on reliability $P(Y<X)$ in the Levy distribution. Mathematical and Computer Modeling, 41(8-9), 965-971.

[13] McIntyre, G. A. (1952): A method of unbiased selective sampling, using ranked sets. Australian Journal of Agricultural Research, 3(4), 385-390.

[14] Meniconi, B. (1995): The power function distribution: A useful and simple distribution to asses electrical component reliability. Microelectronics Reliability, 36(9), 1207-1212.

[15] Meniconi, M. and Barry, D. M. (1996): The power function distribution: A useful and simple distribution to assess electrical component reliability. Microelectronics Reliability, 36(9), 1207-1212.

[16] Muttlak, H. (1997): Median ranked set sampling. Journal of Applied Statistical Science, 6, 245-255.

[17] Saleem, M., Aslam, M., and Economou, P. (2010): On the Bayesian analysis of the mixture of power function distribution using the complete and the censored sample. Journal of Applied Statistics, 37(1), 25-40.

[18] Saran, J. and Pandey, A. (2004): Estimation of parameters of a power function distribution and its characterization by $k$-th record values. Statistica, 14(3), 523-536.

[19] Stokes, S. L. and Sager, T. L. (1988): Characterization of ranked set sample with application to estimating distribution functions. Journal of the American Statistical Association, 83(402), 374-381.

[20] Takahasi, K. and Wakimoto, K. (1968): On unbiased estimates of the population mean based on the sample stratified by means of ordering. Annals of Institute of Statistical Mathematics, 20(1), 1-31. 